\title{
Reference Intervals of Total Testosterone in Adult Filipino Men
}

\author{
Myrna Buenaluz-Sedurante $\mathbb{D}^{1},{ }^{1}$ Mark Isaiah K. Co, ${ }^{2}$ Daryl Jade T. Dagang, ${ }^{2}$ \\ Racquel G. Bruno, ${ }^{2}$ Annie Jane N. Sarmiento, ${ }^{2}$ and Michael L. Tee ${ }^{1}$ \\ ${ }^{1}$ Department of Physiology, University of the Philippines College of Medicine, Manila, Philippines \\ ${ }^{2}$ Division of Endocrinology, Diabetes and Metabolism, Department of Medicine, University of the Philippines College of Medicine, \\ Manila, Philippines \\ Correspondence should be addressed to Myrna Buenaluz-Sedurante; mbsedurante@up.edu.ph
}

Received 24 September 2020; Revised 17 November 2020; Accepted 19 November 2020; Published 29 November 2020

Academic Editor: Vito Angelo Giagulli

Copyright (c) 2020 Myrna Buenaluz-Sedurante et al. This is an open access article distributed under the Creative Commons Attribution License, which permits unrestricted use, distribution, and reproduction in any medium, provided the original work is properly cited.

\begin{abstract}
Background. The reference range of total testosterone needs to be established locally as ethnic differences in adiposity, insulin sensitivity, and sex hormone-binding globulin (SHBG) levels may affect total testosterone levels. The aim of this study is to establish the reference intervals of total testosterone from healthy, young adult Filipino males. Methods. The study included 110 healthy, Filipino male volunteers aged 21-40, studying or working at the University of the Philippines Manila. Clinical history, height, weight, body mass index (BMI), and blood pressure (BP) were obtained, and blood for total testosterone, SHBG, albumin, insulin, fasting blood sugar (FBS), and total cholesterol was collected. Free testosterone was calculated using Vermeulen's formula. The 2.5th to 97.5th percentiles of subjects for total testosterone were used as the normative range for Filipino men. Results. The reference range of total testosterone is $7.33-53.01 \mathrm{nmol} / \mathrm{L}$. Conclusion. The present study derived reference ranges of total testosterone using data from apparently healthy, young adult men to support clinical services.
\end{abstract}

\section{Introduction}

Androgens are vital for the male's physical attributes, distinct strength, behavior, and reproduction [1]. Male androgens mostly come from the testes in the form of testosterone and dihydrotestosterone. The adrenals also contribute a small portion in the form of dehydroepiandrosterone (DHEA) and dehydroepiandrosterone sulfate (DHEA-S). These adrenal hormones can also be converted into testosterone in the periphery. Testosterone levels may decline with age $[2,3]$. In addition, androgen deficiency has been associated with many diseases such as obesity, type 2 diabetes mellitus, metabolic syndrome, depression, and Alzheimer's disease, human immunodeficiency virus (HIV) [4-8] and with the use of certain medications such as steroids, anticonvulsants, and opioids [1]. Androgen deficiency causes osteoporosis, increased cardiovascular mortality, and poor quality of life $[1,9,10]$.

To determine whether a patient is testosterone deficient, guidelines of the European Association of Urology (EAU) and the Endocrine Society (ES) recommend that a clinician considers clinical signs and symptoms in conjunction with two early morning total testosterone values as levels peak in the morning $[11,12]$. If both values are consistently low, then this establishes the diagnosis. If the clinical presentation is associated with a low normal total testosterone, then free testosterone levels should be analyzed via equilibrium dialysis or calculated with the help of sex hormone-binding globulin (SHBG). Recall that, in young adult men, total testosterone is composed of the following fractions: $2 \%$ in the free form, $68 \%$ weakly bound to albumin, and $30 \%$ tightly bound to SHBG [13]. When tightly bound to SHBG, testosterone is not biologically available. Concentrations of SHBG vary widely in healthy men and are related to variables such as diet, body mass index (BMI), insulin concentration, smoking, and age [14]. Thus, ideally, free, and nonspecifically bound testosterone would generally reflect the clinical condition more accurately than total testosterone. However, tests for free (by equilibrium dialysis) and bioavailable testosterone (via ammonium precipitation) are 
nonautomated, are time-consuming, require expensive techniques, and therefore are not routinely performed in most laboratories. Direct radioimmunoassay, while available, has been criticized for the lack of accuracy $[15,16]$. Alternatively, the concentrations of free testosterone and bioavailable testosterone can be calculated by the use of one of several published algorithms. These algorithms assume that when the concentrations of total testosterone, SHBG, and albumin and the constants for the binding of testosterone to SHBG and albumin are known, free testosterone and bioavailable testosterone can be calculated $[17,18]$.

Racial differences in circulating total testosterone levels have been noted due to varying SHBG levels resulting from ethnic differences in adiposity and insulin sensitivity. Thus, normative values for Whites have been determined in many countries such as Germany, the United States, Wales, and Britain [19-23] using various sampling techniques and laboratory methods. So far, no reference values for African Americans have been generated as most studies comparing African Americans and Whites showed that their testosterone levels are not significantly different [24, 25]. Reference values for East Asians have also been published [26, 27]. There are no reference ranges published for Southeast Asians, that is, those who reside in the Philippines, Singapore, Malaysia, Indonesia, and Brunei. This study aims to provide reference data for the definition of normal total testosterone levels in young, healthy, nonobese, Filipino males. In addition, since adiposity decreases insulin sensitivity and this in turn decreases SHBG, total testosterone will be correlated with waist-hip ratio (WHR), SHBG, and insulin levels. The data may then be used to diagnose androgen deficiency among this group.

\section{Methods}

2.1. Study Design. This cross-sectional study was conducted according to a protocol approved by the Technical Review Board of the University of the Philippines, Department of Physiology, and the University of the Philippines Manila Ethics Review Board. This study used the Strengthening the Reporting of Observational Studies in Epidemiology (STROBE) cross-sectional reporting guidelines [28].

2.2. Reference Sample Population. This study invited all healthy, young adult males aged 21-40 years, studying or working at the University of the Philippines Campus in Manila and its affiliated hospital, Philippine General Hospital, from 2016 to 2019 through personal letters of invitation and advertisements. This sampling assumed that healthy members of the said local community adequately represent the population of the country. The sample size was based on studies conducted by Lott and Mitchell [29] and echoed recently by Wellek, et al. [30], which state that a sample size of 110 for normally distributed data and 119 for nonGaussian distributions will result in stable $2.5 \%$ and $97.5 \%$ reference limits at the $95 \%$ confidence level.

The study followed the International Federation of Clinical Chemistry's (IFCC) Expert Panel Recommendations on the Theory of Reference Values which advise a priori selection of participants by exclusion of specific physiologic, pathologic, and lifestyle conditions which contribute to biological variability $[31,32]$. Thus, subjects with the following criteria were excluded from the study: obesity defined as a $\mathrm{BMI} \geq 25$; fasting blood sugar (FBS) $\geq 126 \mathrm{mg} / \mathrm{dl}$; hypertension (BP $\geq 140 / 90 \mathrm{mmHg}$ ); hypercholesterolemia (total cholesterol $\geq 240$ ); self-reported history of diabetes, osteoporosis, chronic lung disease, ulcer, HIV, cancer, cerebrovascular disease, myocardial infarction, stroke, congestive heart failure, bypass, angioplasty, claudication, hyperthyroid or hypothyroid disease, and infertility; current use of prescription medication, including eye drops, topical medications, and inhalers; history and present intake of testosterone, steroids, opioids, anticonvulsants, and male fertility agents; smoking (present or past); alcohol consumption exceeding $600 \mathrm{ml}$ ethanol per week, corresponding to approximately six drinks per day, one drink being $15 \mathrm{ml}$ ethanol (10 oz. beer, $4 \mathrm{oz}$. wine, or $1.5 \mathrm{oz}$. spirits); subject works in shifts; and lastly, a family history of hypogonadism or infertility (self-reported).

\subsection{Data Collection Procedure. Informed consent was ob-} tained after which subjects were interviewed regarding birthdate, past illnesses, current medications, smoking and alcoholic beverage intake, and family illnesses, and then the following were obtained: height, weight, waist circumference, hip circumference, and blood pressure. Height was measured without shoes using a portable stadiometer and recorded to the nearest $0.1 \mathrm{~cm}$. Weight in light clothing without shoes was recorded to the closest $0.1 \mathrm{~kg}$. Waist circumference was measured using a soft measuring tape midway between the lowest rib margin and the iliac crest in a standing position. Hip circumference was measured at the widest part of the gluteal region. Blood pressure was measured using a calibrated aneroid sphygmomanometer. Blood sample was collected in red-top tubes after a 10 hour fast, within 4 hours of awakening.

2.4. Assays. FBS, albumin, and total cholesterol were analyzed using a Cobas Integra 400Plus clinical chemistry analyzer. Total testosterone levels were measured with the Testosterone [I-125] RIA Kit (RK-61CT-Institute of Isotopes Ltd., Budapest). The inter- and intra-assay coefficient of variations $(\mathrm{CV})$ were $12 \%$ and $8.9 \%$, respectively. SHBG levels were determined using the SHBG [I-125] IRMA Kit (RK-86CT-Institute of Isotopes Ltd., Budapest). The interand intra-assay coefficients of variation (CV) were $6.04 \%$ and $8.58 \%$. Lastly, insulin levels were measured using the Insulin [1-125] IRMA Kit (RK-400CT-Institute of Isotopes Ltd., Budapest). The inter- and intra-assay coefficients of variation $(\mathrm{CV})$ were less than $17.1 \%$ and $4.4 \%$, respectively. For the testosterone assay, a zero calibrator, 5 calibrators, and 2 quality control test samples were used, while for the insulin and SHBG assay, the same number of calibrators with 1 quality control test sample was used.

Free testosterone was calculated using the algorithm developed by Vermeulen [17]. This algorithm is available as 
an online calculator provided by the International Society for the Study of the Aging Male (ISSAM) [33].

2.5. Data Analysis. Data were entered in Excel, and descriptive statistics were then calculated. Missing data were managed by case deletion. RefVal 4.11 (Solberg, Oslo, Norway), a program developed by the IFCC to calculate reference intervals, was used [34]. The program defines the reference interval as the interval of values containing the central $95 \%$ of a healthy population. As part of the program, nonnormally distributed data are transformed. The two-stage transformation utilizes the Manly exponential transformation to remove skewness as its first stage and the modulus function of John and Draper as the final stage to remove the remaining kurtosis of the distribution. If the normality assumptions are still not met after the transformation, a nonparametric bootstrap method is employed where reference intervals are estimated over large numerous resamples. For this study, the program calculated reference intervals parametrically. Data are expressed as mean \pm SD, median, and range at 2.5th to 97.5th percentiles.

In addition to using RefVal, nonparametric correlation tests using Spearman's rank rho coefficient were used to assess the association between variables.

\section{Results}

A total of 190 males volunteered to participate in the study, but 71 were excluded due to the history of smoking and increased BMI. One hundred and nineteen were eventually enrolled. Five participants were excluded due to incomplete data, while 4 were excluded due to increased FBS or cholesterol. The final study sample included 110 males with age between 21 and 40. The mean age and SD were 27.49 and 5.33 years. Anthropometric measures showed a mean and SD of BMI of 22.32 and $1.96 \mathrm{~kg} / \mathrm{m}^{2}$ and a waist-hip ratio (WHR) of 0.92 and 0.05 . Table 1 presents the demographic profile and anthropometric profile of these healthy, young adult Filipino males. The reference range for total testosterone is $7.33-53.01 \mathrm{nmol} / \mathrm{L}$, with a mean, $\mathrm{SD}$ of testosterone of $22.68 \mathrm{nmol} / \mathrm{L}, 12.37$ and a median of $18.61 \mathrm{nmol} / \mathrm{L}$. Calculated free testosterone showed a mean, SD of $0.55 \mathrm{nmol} / \mathrm{L}, 0.33$ with a median of $0.44 \mathrm{nmol} / \mathrm{L}$. Regarding distribution, total testosterone levels were skewed to the right. The serum insulin levels were also skewed to the right with an evident outlier that was also detected by Dixon's algorithm. The distribution of sex hormone-binding globulin concentration was almost symmetrical. After transformation, total testosterone, insulin, and SHBG levels assumed a Gaussian distribution. See Supplementary Figures S1-S6 in Supplementary Materials for full image analysis.

The correlation between total testosterone, insulin, WHR, and SHBG is shown in Table 2. Insulin was positively correlated with WHR and negatively correlated with SHBG. Total testosterone was positively correlated with SHBG.
TABLE 1: Characteristics of study participants.

\begin{tabular}{|c|c|c|}
\hline & Mean & Standard deviation \\
\hline Age (years) & 27.49 & 5.33 \\
\hline Height (m) & 168.59 & 6.37 \\
\hline Weight (kg) & 63.74 & 7.62 \\
\hline $\mathrm{BMI}^{\mathrm{a}}\left(\mathrm{kg} / \mathrm{m}^{2}\right)$ & 22.32 & 1.96 \\
\hline Waist-hip ratio & 0.92 & 0.05 \\
\hline $\mathrm{SBP}^{\mathrm{b}}(\mathrm{mmHg})$ & 115.38 & 10.93 \\
\hline $\mathrm{DBP}^{\mathrm{c}}(\mathrm{mmHg})$ & 73.21 & 8.63 \\
\hline $\mathrm{FBS}^{\mathrm{d}}(\mathrm{mg} / \mathrm{dl})$ & 86.29 & 7.46 \\
\hline Total cholesterol (mg/dl) & 179.91 & 35.53 \\
\hline Albumin $(\mathrm{g} / \mathrm{l})$ & 49.21 & 2.44 \\
\hline Insulin (mIU/ml) & 11.08 & 8.9 \\
\hline HOMA2 $\mathrm{IR}^{\mathrm{e}}$ & 1.36 & 0.85 \\
\hline $\mathrm{SHBG}^{\mathrm{f}}(\mathrm{nmol} / \mathrm{L})$ & 24.46 & 10.6 \\
\hline
\end{tabular}

${ }^{\mathrm{a} B o d y}$ mass index; ${ }^{\mathrm{b}}$ systolic blood pressure; ${ }^{\mathrm{c}}$ diastolic blood pressure; ${ }^{\mathrm{d}}$ fasting blood sugar; ${ }^{\mathrm{e}}$ homeostatic model assessment of insulin resistance; fex hormone-binding globulin.

\section{Discussion}

We developed reference ranges for young, nonobese Filipino males aged 21-40. There are no reported reference intervals for other predominantly Malay groups such as those from Indonesia, Thailand, Brunei, nor Singapore, although there is an article comparing average testosterone levels between Malays and Chinese men in Malaysia using chemiluminescence enzyme immunoassay (CLEIA) [35]. This study written by Chin and colleagues reported a mean of $20.4 \mathrm{nmol} / \mathrm{L}$ with a standard deviation of $6.4 \mathrm{nmol} / \mathrm{L}$. This is comparable with our results, with a mean of $21.86 \mathrm{nmol} / \mathrm{L}$ and a standard deviation of $10.8 \mathrm{nmol} / \mathrm{L}$. Table 3 is a comparison of reference ranges for various ethnic groups. The lower limit of our study's reference range is closest to that established by Iwamoto et al. for the Japanese cohort [27]. Our results also span the range of normative values for most ethnic groups.

Increased adiposity causes insulin resistance resulting in a subsequent rise in insulin levels. Insulin resistance decreases SHBG levels resulting in lower total testosterone levels [14]. This is echoed by our study where insulin levels were positively correlated with WHR and negatively correlated with SHBG. However, insulin was not negatively correlated with total testosterone as might have been expected. To further investigate this last analysis, we then calculated Homeostatic Model Assessment of Insulin Resistance (HOMA IR), a standard measure of insulin resistance, and correlated it with total testosterone. This post hoc analysis did not reveal a significant correlation either, probably due to small sample size. Lastly, the study also showed that total testosterone and SHBG were positively correlated.

Our study has several strengths. First, stringent criteria were used to select subjects, excluding the obese, smokers, and diseases or conditions which may affect total testosterone levels. Second, a single technician using meticulous quality control procedures performed the assays.

The study has several limitations. First, our study sample is not population-based but instead tested healthy volunteers. However, the ICC deems samples from both groups as 
TABLE 2: Spearman's rho correlation between testosterone, SHBG, and insulin levels.

\begin{tabular}{lcccc}
\hline$X$ & $Y$ & Spearman's rho & $p$ value & Correlation \\
\hline Insulin & WHR & 0.27 & 0.004 & Weak positive \\
Insulin & SHBG & -0.36 & 0.001 & Moderate negative \\
Insulin & Total testosterone & 0.08 & 0.392 & Insignificant \\
Total testosterone & HOMA2 IR $^{\mathrm{a}}$ & 0.08 & 0.419 & Insignificant \\
Total testosterone & SHBG $^{\mathrm{b}}$ & 0.21 & 0.024 & Weak positive \\
\hline
\end{tabular}

${ }^{\mathrm{a}}$ Homeostatic model assessment of insulin resistance; ${ }^{\mathrm{b}}$ sex hormone-binding globulin.

TABLE 3: Reference ranges for total testosterone in young adult males across different ethnic groups.

\begin{tabular}{|c|c|c|c|c|}
\hline Study author & Race & Assay & $N$ & $\begin{array}{l}95 \% \text { range } \\
(\mathrm{nmol} / \mathrm{l})\end{array}$ \\
\hline \multicolumn{5}{|c|}{ Population-based } \\
\hline $\begin{array}{l}\text { Friedrich } \\
2008(20)\end{array}$ & $\begin{array}{c}\text { White } \\
\text { (Germany) }\end{array}$ & CLEIA $^{\mathrm{a}}$ & 416 & $10.4-32.3$ \\
\hline $\begin{array}{l}\text { Travison } \\
2017(24)\end{array}$ & $\begin{array}{c}\text { White } \\
\text { (US and Europe) }\end{array}$ & $\mathrm{LC}^{-\mathrm{MS}^{\mathrm{b}}}$ & 400 & $9.16-31.78$ \\
\hline $\begin{array}{l}\text { Shen } \\
2013(26)\end{array}$ & $\begin{array}{l}\text { East Asian } \\
\text { (China) }\end{array}$ & CLEIA & 227 & $13.61-19.28$ \\
\hline $\begin{array}{l}\text { Iwamoto } \\
2004(27)\end{array}$ & $\begin{array}{c}\text { East Asian } \\
\text { (Japan) }\end{array}$ & RIA & 1172 & $6.96-26.00$ \\
\hline \multicolumn{5}{|c|}{ Healthy volunteers } \\
\hline $\begin{array}{l}\text { Shatzl } \\
2003(21)\end{array}$ & $\begin{array}{c}\text { White } \\
\text { (Austria) }\end{array}$ & CLEIA & 133 & $10.4-28.8$ \\
\hline $\begin{array}{l}\text { Boyce } \\
2004(22)\end{array}$ & $\begin{array}{l}\text { White } \\
\text { (UK) }\end{array}$ & $\mathrm{RIA}^{\mathrm{c}}$ & 199 & $10.04-38.76$ \\
\hline $\begin{array}{l}\text { Elmlinger } \\
2005,(23)\end{array}$ & $\begin{array}{c}\text { White } \\
\text { (Germany) }\end{array}$ & CLEIA & 446 & $8.7-29.6$ \\
\hline $\begin{array}{l}\text { Neale } \\
2013(24)\end{array}$ & $\begin{array}{l}\text { White } \\
\text { (Wales) }\end{array}$ & LC-MS & 67 & $10.6-31.9$ \\
\hline
\end{tabular}

${ }^{\mathrm{a} C h e m i l u m i n e s c e n c e ~ e n z y m e ~ i m m u n o a s s a y ; ~}{ }^{\mathrm{b}}$ liquid chromatography-mass spectrometry; ${ }^{c}$ radioimmunoassay.

valuable and acceptable. If we look at the population-based compared to healthy volunteer studies in Whites, as tabulated in Table 3, note that the 2 population-based studies, the first by Freidrich and the second by Travison, both showed comparable results with three of the four nonpopulation-based studies [21-24]. Incidentally, note also that the results were similar despite differing laboratory techniques (CLEIA and LSMS). Second, we generated reference intervals for young adult men only following the approach of Bhasin and colleagues [36]. Reference intervals are generated from this age group for parameters that exhibit substantial age-related changes such as $T$-scores in bone mineral density and reference values for muscle strength and physical performance in sarcopenia.

In conclusion, we generated reference ranges for total testosterone specific for Filipinos using data from apparently healthy men.

\section{Data Availability}

The data that support the findings of this study are available from the corresponding author upon reasonable request.

\section{Conflicts of Interest}

The authors declare no conflicts of interest.

\section{Acknowledgments}

This study was funded through a grant from the National Institutes of Health under the Project Code NIH 2016-005. The authors thank Patrick Wincy Reyes for the statistical analysis of this study.

\section{Supplementary Materials}

Figure 1: distribution of nontransformed data for total testosterone. Figure 2: distribution of nontransformed data for serum insulin. Figure 3: distribution of nontransformed data for sex hormone-binding globulin. Figure 4: distribution of transformed data for total testosterone. Figure 5: distribution of transformed data for serum insulin. Figure 6: distribution of transformed data for sex hormone-binding globulin. http://downloads.hindawi.com/journals/ije/2020/ 8877261.f1.zip. (Supplementary Materials)

\section{References}

[1] S. Basaria, "Male hypogonadism," The Lancet, vol. 383, no. 9924, pp. 1250-1263, 2014.

[2] J. B. Shelton and J. Rajfer, "Androgen deficiency in aging and metabolically challenged men," Urologic Clinics of North America, vol. 39, no. 1, pp. 63-75, 2012.

[3] M. J. Diver, K. E. Imtiaz, A. M. Ahmad, J. P. Vora, and W. D. Fraser, "Diurnal rhythms of serum total, free and bioavailable testosterone and of SHBG in middle-aged men compared with those in young men," Clinical Endocrinology, vol. 58, no. 6, pp. 710-717, 2003.

[4] D. M. Kelly and T. H. Jones, "Testosterone and obesity," Obesity Reviews, vol. 16, no. 7, pp. 581-606, 2015.

[5] F. W. Gibb and M. W. J. Strachan, "Androgen deficiency and type 2 diabetes mellitus," Clinical Biochemistry, vol. 47, no. 10-11, pp. 940-949, 2014.

[6] O. P. Almeida, A. Waterreus, N. Spry, L. Flicker, and R. N. Martins, "One year follow-up study of the association between chemical castration, sex hormones, beta-amyloid, memory and depression in men," Psychoneuroendocrinology, vol. 29, no. 8, pp. 1071-1081, 2004.

[7] S. D. Moffat, A. B. Zonderman, E. J. Metter et al., "Free testosterone and risk for Alzheimer disease in older men," Neurology, vol. 62, no. 2, pp. 188-193, 2004.

[8] V. Rochira and G. Guaraldi, "Hypogonadism in the HIVinfected man," Endocrinology and Metabolism Clinics of North America, vol. 43, no. 3, pp. 709-730, 2014.

[9] M. Almeida, M. R. Laurent, V. Dubois et al., "Estrogens and androgens in skeletal physiology and pathophysiology," Physiological Reviews, vol. 97, no. 1, pp. 135-187, 2017.

[10] D. Chistiakov, V. Myasoedova, A. Melnichenko, A. Grechko, and A. Orekhov, "Role of androgens in cardiovascular 
pathology," Vascular Health and Risk Management, vol. 14, pp. 283-290, 2018.

[11] G. R. Dohle, S. Arver, C. Betocchi, T. H. Jones, and S. Kliesch, "EAU 2018 guideline on male hypogonadism," 2018, http:// uroweb.org/guideline/malehypogonadism.

[12] S. Bhasin, J. P. Brito, G. R. Cunningham et al., "Testosterone therapy in men with hypogonadism: an endocrine society* clinical practice guideline," The Journal of Clinical Endocrinology \& Metabolism, vol. 103, no. 5, pp. 1715-1744, 2018.

[13] J. F. Dunn, B. C. Nisula, and D. Rodbard, "Transport of steroid hormones: binding of 21 endogenous steroids to both testosterone-binding globulin and corticosteroid-binding globulin in human plasma," The Journal of Clinical Endocrinology \& Metabolism, vol. 53, no. 1, pp. 58-68, 1981.

[14] A. Vermeulen, J. M. Kaufman, and V. A. Giagulli, "Influence of some biological indexes on sex hormone-binding globulin and androgen levels in aging or obese males," Journal of Clinical Endocrinology \& Metabolism, vol. 81, no. 5, pp. 1821-1826, 1996.

[15] K. Van Uytfanghe, D. Stöckl, J. M. Kaufman, T. Fiers, A. De Leenheer, and L. M. Thienpont, "Validation of 5 routine assays for serum free testosterone with a candidate reference measurement procedure based on ultrafiltration and isotope dilution-gas chromatography-mass spectrometry," Clinical Biochemistry, vol. 38, no. 3, pp. 253-261, 2005.

[16] S. M. Neale, R. Hocking, M. Biswas et al., "Adult testosterone and calculated free testosterone reference ranges by tandem mass spectrometry," Annals of Clinical Biochemistry, vol. 50, no. 2, pp. 159-161, 2013.

[17] A. Vermeulen, L. Verdonck, and J. M. Kaufman, “A critical evaluation of simple methods for the estimation of free testosterone in serum," The Journal of Clinical Endocrinology \& Metabolism, vol. 84, no. 10, pp. 3666-3672, 1999.

[18] W. De Ronde, Y. T. Van Der Schouw, H. A. Pols et al., "Calculation of bioavailable and free testosterone in men: a comparison of 5 published algorithms," Clinical Chemistry, vol. 52, no. 9, pp. 1777-1784, 2006.

[19] N. Friedrich, H. Völzke, D. Rosskopf et al., "Reference ranges for serum dehydroepiandrosterone sulfate and testosterone in adult men," Journal of Andrology, vol. 29, no. 6, pp. 610-617, 2008.

[20] G. Shatzl, S. Madersbacher, C. Temml et al., "Serum androgen levels in men: impact of health status and age," Urology, vol. 61, no. 3, pp. 629-633, 2003.

[21] M. J. Boyce, K. J. Baisley, E. V. Clark, and S. J. Warrington, "Are published normal ranges of serum testosterone too high? Results of a cross-sectional survey of serum testosterone and luteinizing hormone in healthy men," BJU International, vol. 94, no. 6, pp. 881-885, 2004.

[22] M. W. Elmlinger, W. Kühnel, H. Wormstall, and P. C. Döller, "Reference intervals for testosterone, androstenedione and SHBG levels in healthy females and males from birth until old age," Clinical Laboratory, vol. 51, no. 11-12, pp. 625-632, 2005.

[23] T. G. Travison, H. W. Vesper, E. Orwoll et al., "Harmonized reference ranges for circulating testosterone levels in men of four cohort studies in the United States and Europe," The Journal of Clinical Endocrinology \& Metabolism, vol. 102, no. 4, pp. 1161-1173, 2017.

[24] H. J. Litman, S. Bhasin, C. L. Link, A. B. Araujo, and J. B. McKinlay, "Serum androgen levels in black, hispanic, and white men," The Journal of Clinical Endocrinology \& Metabolism, vol. 91, no. 11, pp. 4326-4334, 2006.
[25] A. Richard, S. Rohrmann, L. Zhang et al., "Racial variation in sex steroid hormone concentration in black and white men: a meta-analysis," Andrology, vol. 2, no. 3, pp. 428-435, 2014.

[26] X. Shen, R. Wang, N. Yu et al., "Correction: reference ranges and association of age and lifestyle characteristics with testosterone, sex hormone binding globulin, and luteinizing hormone among 1166 western Chinese men," PLoS One, vol. 11, no. 12, Article ID e0168029, 2016.

[27] T. Iwamoto, T. Yanase, E. Koh et al., "Reference ranges of serum total and free testosterone in Japanese male adults," The Japanese Journal of Urology, vol. 95, no. 6, pp. 751-760, 2004.

[28] E. Von Elm, D. G. Altman, M. Egger, S. J. Pocock, P. C. Gøtzsche, and J. P. Vandenbroucke, "The strengthening the reporting of observational studies in epidemiology (STROBE) statement: guidelines for reporting observational studies," Journal of Clinical Epidemiology, vol. 61, no. 4, pp. 344-349, 2008.

[29] J. A. Lott, L. C. Mitchell, M. L. Moeschberger, and D. E. Sutherland, "Estimation of reference ranges: how many subjects are needed?" Clinical Chemistry, vol. 38, no. 5, pp. 648-650, 1992.

[30] S. Wellek, K. J. Lackner, C. Jennen-Steinmetz, I. Reinhard, I. Hoffmann, and M. Blettner, "Determination of reference limits: statistical concepts and tools for sample size calculation," Clinical Chemistry and Laboratory Medicine (CCLM), vol. 52, no. 12, pp. 1685-1694, 2014.

[31] H. E. Solberg, "International federation of clinical chemistry (IFCC), scientific committee, clinical section, Expert Panel on theory of reference values, and international committee for standardization in haematology (ICSH), standing committee on reference values. Approved recommendation (1986) on the theory of reference values. Part 1 . The concept of reference values," Journal of clinical chemistry and clinical biochemistry, vol. 25, no. 5, pp. 337-342, 1987.

[32] International Federation of Clinical Chemistry (IFCC), "Scientific Committee, Clinical Section. Expert Panel on Theory of Reference Values (EPTRV). IFCC Document (1982) stage 2, draft 2, 1983-10-07 with a proposal for an IFCC recommendation. The theory of reference values. Part 2 . Selection of individuals for the production of reference values," Clinica Chimica Acta, vol. 139, no. 2, pp. 205F-213F, 1984.

[33] http://www.issam.ch/freetesto.htm.

[34] H. E. Solberg, "RefVal: a program implementing the recommendations of the International Federation of Clinical Chemistry on the statistical treatment of reference values," Computer Methods and Programs in Biomedicine, vol. 48, no. 3, pp. 247-256, 1995.

[35] K. Y. Chin, I. N. Soelaiman, I. N. Mohamed, H. Joharj, and W. Z. Wan Ngah, "Ethnicity, smoking and body composition influence testosterone and estradiol levels in healthy young adult men in Malaysia: a pilot study," International Journal of Endocrinology \& Metabolism, vol. 10, no. 1, pp. 404-410, 2012.

[36] S. Bhasin, M. Pencina, G. K. Jasuja et al., "Reference ranges for testosterone in men generated using liquid chromatography tandem mass spectrometry in a community-based sample of healthy nonobese young men in the Framingham Heart Study and applied to three geographically distinct cohorts," The Journal of Clinical Endocrinology \& Metabolism, vol. 96, no. 8, pp. 2430-2439, 2011. 